\title{
Ultraviolet broadband light scattering for optically-trapped submicron-sized aerosol particles
}

\section{Journal Article}

Author(s):

David, Grégory; Esat, Kivanç (1D; Ritsch, Irina; Signorell, Ruth (1)

Publication date:

2016

Permanent link:

https://doi.org/10.3929/ethz-b-000113784

Rights / license:

In Copyright - Non-Commercial Use Permitted

Originally published in:

Physical Chemistry Chemical Physics 18(7), https://doi.org/10.1039/c5cp06940h

Funding acknowledgement:

159205 - Interaction of Light with Ultrafine Aerosol Particles II (SNF) 


\title{
Ultraviolet broadband light scattering for optically-trapped submicron- sized aerosol particles $\dagger$
}

Grégory David, Kıvanç Esat, Irina Ritsch, and Ruth Signorell*

Laboratory of Physical Chemistry, ETH Zurich, Vladimir-Prelog-Weg 2, CH-8093, Zurich, Switzerland

\begin{abstract}
We describe a broadband light scattering setup for the characterization of size and refractive index of single submicron-to-micron sized aerosol particles. Individual particles are isolated in air by a quadruple Bessel beam optical trap or a counter-propagating optical tweezer. The use of very broadband radiation in the wavelength range from 320 to $700 \mathrm{~nm}$ covering the ultraviolet region allows to size submicron particles. We show that a broad wavelength range is required to determine the particle radius and the refractive index with an uncertainty of several nanometers and $\sim 0.01$, respectively. The smallest particle radius that can be accurately determined lies around $300 \mathrm{~nm}$. Wavelength-dependent refractive index data over a broad range are obtained, including the ultraviolet region where corresponding data are rare. Four different applications are discussed: 1) the sizing of submicron PSL spheres, 2) the evaporation of binary glycerol water droplets, 3) hydration/dehydration cycling of aqueous potassium carbonate droplets, and 4) photochemical reactions of oleic acid droplets.
\end{abstract}

Table of content sentence: A broadband light scattering experiment for the characterization of size and refractive index of single submicron-to-micron sized aerosol particles is presented.

*rsignorell@ethz.ch

+ Electronic Supplementary Information (ESI) available. See DOI: 10.1039/x0xx00000x 


\section{Introduction}

Size, shape, and refractive index are the key properties of aerosol particles that govern their interaction with sunlight 1,2 and hence their direct climate impact ${ }^{3}$. These quantities are needed to accurately simulate light-particle interaction ${ }^{1,4,5}$, to analyse laboratory ${ }^{6-8}$ and atmospheric ${ }^{9}, 10$ light scattering measurements, and to properly incorporate light-particle interaction into climate modelling ${ }^{2}$. Atmospheric processes, including evaporation ${ }^{11}$, hygroscopic growth ${ }^{12}$ and aging ${ }^{13}$, constantly alter the properties of atmospheric aerosols. Laboratory studies of individual particles play an important role in this context since they allow detailed investigations of physicochemical properties and processes by avoiding ensemble averaging over multiple sizes, shapes, and optical properties ${ }^{11,13-15}$.

Size and refractive index of an isolated single aerosol particle can be determined by a variety of experiments using different types of traps for the isolation of single particles in the gas phase ${ }^{11,16-19}$. Some of these provide refractive indices only at selected wavelengths ${ }^{11,19}$. Broadband cavity enhanced spectroscopy (BBCES) provides the real and imaginary part of the refractive index over a somewhat broader wavelength range (from $\sim 360$ to $420 \mathrm{~nm})^{20}$. However, to the best of our knowledge BBCES measurements have only been demonstrated for particle ensembles and to accurately retrieve the refractive index as a function of the wavelength these measurements need to be performed on mono-disperse aerosol particles for multiple sizes ${ }^{20}$. Other single particle measurements, which we refer to as Broadband Light Scattering (BLS) measurements, provide refractive indices $m(\lambda)$ over a range of different wavelengths $\lambda^{17,18,21-25}$. The range of wavelengths studied varies widely (from 543 to 680 in ${ }^{16}$, from 560 to $610 \mathrm{in}^{17}$, from 480 to $700 \mathrm{~nm}$ in ${ }^{21}$, from 430 to $490 \mathrm{~nm}$ in ${ }^{23}$, from 630 to 662 $\mathrm{nm}$ in ${ }^{24}$ and from 380 to $750 \mathrm{~nm}$ in ${ }^{8}$ ), but has so far been restricted to the visible. An intriguing feature of BLS spectra is the possibility to retrieve single particle size and refractive index simultaneously. This makes BLS measurements well suited to probe the evolution of size and refractive index, e.g. during evaporation, hygroscopic growth or aging. One of the current limitations of BLS experiments is the smallest particle size accessible. To the best of our knowledge, particles smaller than $940 \mathrm{~nm}^{25}$ in radius have never been detected by BLS. However, these submicron-sized particles (also referred to as fine particles ${ }^{26}$ ) are of high atmospheric relevance because of their higher abundance and long residential time in the atmosphere ${ }^{27}$. In general, they play a key role in the direct and indirect effects of aerosols on climate (absorption and scattering of the solar radiation and modification of cloud coverage 
and lifetime because aerosol particle act as cloud condensation nuclei $(\mathrm{CCN})$, respectively) ${ }^{26}$, 28 . Therefore, the study of submicron-sized aerosol particles is of crucial importance.

The present paper introduces a BLS setup that allows us to access the submicrometer size range and to simultaneously determine size and refractive index. We combine a very broadband light source for scattering measurements between $230 \mathrm{~nm}$ and $2000 \mathrm{~nm}$ with either a quadruple Bessel beam (QBB) optical trap or a counter-propagating optical tweezer $(\mathrm{CPT})^{8}$, 23, 29-31. These types of optical traps allow for stable trapping of submicron aerosol particles with tight particle confinement (better than $100 \mathrm{~nm}^{29}$ ) providing optimal conditions for light scattering measurements. The inclusion of the ultraviolet (UV) range of the electromagnetic spectrum is not only crucial for the sizing of submicron particles, it also allows the retrieval of refractive indices in a range where such data are rare but urgently needed. Section 2 describes the experimental BLS setup. Section 3 discusses the important factors that determine the uncertainty in the retrieved particle size and refractive index. Finally, Section 4 presents some applications.

\section{Experiment and data analysis}

\subsection{Experimental setup}

The setup of the $\mathrm{QBB}^{8,29}$ and $\mathrm{CPT}^{23,30,31}$ traps is explained in the Electronic Supplementary Information (ESI $\dagger$; Section A, Fig. A). The principle of single particle BLS measurements has been detailed elsewhere ${ }^{16-18,21-25}$ so only a brief explanation is provided here. The intensity of the light scattered by a single particle is recorded as a function of the wavelength $\lambda$. Typically, only the peak positions of the morphologically dependent resonances (MDR) ${ }^{16}$, 17,32 (also called whispering gallery modes ${ }^{24}$ or Mie resonances ${ }^{18}$ for spherical particles) are used to refine the particle radius $\mathrm{R}$ (for spheres) and the wavelength dependent refractive index $m(\lambda)$. The position and the intensity profile of the MDRs depends on the particle shape, the refractive index, and the size parameter $X=2 \pi R / \lambda$. It is convenient to use $X$ because the particle-light interaction depends on the ratio of particle radius and wavelength. For micronsize particles and broadband light in the visible (VIS) range; i. e. for larger X, the intensity of the scattered light is strong and many sharp resonances can be exploited in the fitting procedure. Performing BLS measurements on submicron particles in the VIS range is challenging because $\mathrm{X}$ is much smaller. As a consequence, the number of resonances in a certain wavelength interval is substantially reduced (for very small particles they disappear 
completely) and the resonances broaden ${ }^{1}$. Furthermore, the intensity of the scattered light is much lower for small particles resulting in reduced sensitivity.

Our BLS setup (Fig. 1) takes advantage of the shorter wavelengths in the UV spectral range to partially compensate for the smaller radius of submicron spheres $(X=2 \pi R / \lambda)$, hence allowing the sizing of submicron particles. In addition, light collection is optimized to improve the sensitivity. A broadband fiber coupled Xe lamp (Ocean Optics, HPX-2000-HPDUV) is used as the light source. It emits wavelengths between $230 \mathrm{~nm}$ and $2000 \mathrm{~nm}$. However, the best BLS results are obtained in the range between 320 to $700 \mathrm{~nm}$. Note that this range is still much broader than in previous setups 16, 17, 21, 23, 24. The lower limit is determined by the low light intensity in the deeper UV, which is more than an order of magnitude lower than in the UV and visible spectral range. For wavelengths longer than 700 $\mathrm{nm}$, several strong emission lines in the lamp spectrum partially hinder the acquisition of accurate data and this range typically does not provide a lot of additional information. The light generated by the fibre coupled Xe lamp is collimated with a reflective collimator (Thorlabs, RC04SMA-F01) and focused onto the trapped particle with a reflective objective (Davin Optronics, 5004-190, numerical aperture (NA) $=0.5$, working distance $(\mathrm{WD})=8.6 \mathrm{~mm})$. The focusing and collimating optics are reflective to minimize chromatic aberrations. The influence of chromatic aberrations and their correction are discussed in the ESI $\uparrow$. Reflective optics provide nearly constant reflectivity between 230 and $1000 \mathrm{~nm}$. However, reflective objectives usually induce more spherical aberrations than transmitting objectives, which might slightly decrease the signal to noise ratio. To avoid collecting scattered light from the trapping laser at $532 \mathrm{~nm}$ optical filters are used. The light scattered by the trapping laser is roughly five orders of magnitude more intense than the BLS. Since optical filters with sufficient optical density (OD) that block $532 \mathrm{~nm}$ light but transmit wavelengths from 320 to $700 \mathrm{~nm}$ are not commercially available we used two different optical filters: A shortpass filter (Edmund optics, 49825, optical density OD $>4$ ) for the wavelengths range from 320 to $500 \mathrm{~nm}$ and a notch filter (Edmund optics, 86120, OD 6) for the ranges from 380 to $515 \mathrm{~nm}$ and from 550 to $700 \mathrm{~nm}$. The BLS is collected over a range of scattering angles $\theta$ between $73.7^{\circ}<\theta<106.3^{\circ}$ and collimated with a second reflective objective (Davin Optronics, 5001-190, NA $=0.28, \mathrm{WD}=24.5 \mathrm{~mm}$ ). The scattered light is collected around $\theta \approx$ $90^{\circ}$ and not in backward direction to avoid strong background contributions arising from the partial back-reflection of the reflective objective and the fused quartz windows of the trapping cell. The collected BLS light is then focused with a reflective collimator (Thorlabs, RC04FC- 
P01) into the $600 \mu \mathrm{m}$ diameter solarisation resistant fibre of the spectrometer (Ocean Optics, Maya2000 PRO with a longpass order filter, 200-1100 nm, $25 \mu \mathrm{m}$ slit, $1.1 \mathrm{~nm}$ resolution) and recorded by a CCD camera. Typical integration times per spectrum vary between 150 to 2000 ms, depending on the size of the particle.

BLS measurements are performed on single glycerol particles (Sigma-Aldrich, $\geq 99 \%$ ), aqueous $\left(\mathrm{H}_{2} \mathrm{O}\right.$, Merck Millipore, Lichrosolv $\left.{ }^{\circledR}\right)$ potassium carbonate particles $\left(\mathrm{K}_{2} \mathrm{CO}_{3}\right.$, Acros, $>99 \%$ ), oleic acid particles (Sigma-Aldrich, $\geq 99 \%$ ) and polystyrene latex spheres (PSL) with radii of $509.5 \pm 7.5 \mathrm{~nm}$ (Duke Standarts ${ }^{\mathrm{TM}}$, 4010A, Lot: 43189) and $1550 \pm 145 \mathrm{~nm}$ (Molecular Probes ${ }^{\circledR}$, S37223, Lot: 1620236), respectively. The particles are generated from liquid glycerol, aqueous $\mathrm{K}_{2} \mathrm{CO}_{3}$ solutions $\left(1 \mathrm{~mol} \cdot \mathrm{L}^{-1}\right)$, liquid oleic acid, and aqueous solution containing PSL spheres with a medical nebulizer (PARI LC SPRINT Nebulizer) using nitrogen gas (Pangas, nitrogen 5.0, $\geq 99.999 \%$ ). Single aerosol particles are captured in the optical trap from the plume of particles generated by the nebulizer. A mixed flow of dry and humidified nitrogen is used to control the relative humidity $(\mathrm{RH})$ in the trapping cell. The flow is regulated using two independent flowmeters (Bronkhost, EL-FLOW®, F-201CV). A dry nitrogen flow is used to dry the nebulized PSL particles. The temperature and RH in the cell are measured with two sensors (Honeywell HIH-4602 A) placed at the inlet and at the outlet of the flow.

\section{Fitting parameters and accuracy of size and refractive index determination}

The experimental BLS data are analyzed using a least squares fitting procedure to determine best estimates for the particle radius $R$ and the refractive index $m(\lambda)$ as described in detail in the ESI $\uparrow$. For the sake of computational efficiency previous contributions have usually considered only the resonance peak positions. This limitation can significantly impair the reliability of the analysis (see ESI $\dagger$ ). To avoid this problem we follow a different approach, which exploits the complete information contained in resonance positions, intensities, and lineshapes, by fitting the whole BLS spectrum. We represent the refractive index in terms of the Cauchy parameters $\mathbf{M}=\left\{\mathrm{m}_{0}, \mathrm{~m}_{1} \cdot \mathrm{m}_{2}\right\}$, defined through the Cauchy expansion ${ }^{21,33}$, 


$$
m(\lambda)=m_{0}+m_{1}\left(\frac{\lambda_{0}^{2}}{\lambda^{2}}-1\right)+m_{2}\left(\frac{\lambda_{0}^{4}}{\lambda^{4}}-1\right)
$$

The refractive index at some reference wavelength $\lambda_{0}$ is taken as the non-dispersive contribution $\mathrm{m}_{0}=\mathrm{m}\left(\lambda_{0}\right)$, while $\mathrm{m}_{1}$ and $\mathrm{m}_{2}$ describe the dispersive part of $\mathrm{m}(\lambda)$. Results are quoted with absolute uncertainties for a $95 \%$ confidence interval $\Delta$ (see ESI $\uparrow$, Section B.3. for details). In the following we discuss the pertinent aspects of our analysis in more detail: Section 3.1 addresses the issue of correlation between the fit parameters (R,M) and the influence of the wavelength range included in the fit on their absolute uncertainties. In Section 3.2 we discuss the advantage of a dispersion relation referred to a reference wavelength, Eq.(1), over other formulations ${ }^{18}$. If not otherwise stated we set $m_{2}=0$. How this affects the fit is also considered in Section 3.2. Although beyond the scope of the present paper, we briefly discuss the determination of the imaginary part $\mathrm{k}$ of the complex index of refraction from BLS measurements.

\subsection{Correlation between the fit parameters and wavelength range}

Fig. 2 shows two-dimensional cuts through the sum of squared deviations (SSQ) (ESI $\dagger$ Eq. (B.3)) as a function of the fit parameters $(\mathrm{R}, \mathbf{M})$ for a size-calibrated PSL bead with radius $\mathrm{R}=$ $1550 \pm 145 \mathrm{~nm}$ (width of the size distribution as quoted by the manufacturer) over the wavelength interval $\lambda \epsilon[320 \mathrm{~nm}, 500 \mathrm{~nm}]$. Multiple minima are clearly visible with long flat valleys indicating strong correlations between individual parameters. The strong correlation between $\mathrm{R}$ and $\mathrm{m}_{0}$, for example, means that a smaller radius can partially compensate a larger index of refraction without significantly increasing SSQ, as can be seen in Fig. 2a. The statistical analysis yields the following correlation matrix for the parameter set $\left(\mathrm{R}, \mathrm{m}_{0}, \mathrm{~m}_{1}\right)$ $($ ESI $\dagger$ Eq. (B.6)).

$$
\mathbf{F}_{\text {corr }}=\left(\begin{array}{ccc}
1 & -0.85 & 0.26 \\
-0.85 & 1 & -0.60 \\
0.26 & -0.60 & 1
\end{array}\right)
$$

This example is typical for the analysis of BLS experiments. $\mathrm{R}$ and $\mathrm{m}_{0}$ are strongly anticorrelated (Fig. 2a), $\mathrm{m}_{1}$ and $\mathrm{m}_{0}$ are partially anti-correlated (Fig. 2b), and $\mathrm{R}$ and $\mathrm{m}_{1}$ are weakly correlated (Fig. 2c). In some cases, $\mathrm{m}_{1}$ and $\mathrm{m}_{0}$ are correlated and $\mathrm{R}$ and $\mathrm{m}_{1}$ are anti-correlated. However, the absolute strength of the correlations remains approximately the same as in this example. It is not possible to avoid such correlations, which make a major contribution to the 
statistical uncertainty of the parameters. Fig. 2c also shows multiple local minima in SSQ for different sets of parameters (R,M). Such multiple local minima with similar SSQ values can also cause large uncertainties in the absolute values of $R$ and $m(\lambda)$. Both effects, the correlations between the fit parameters and the existence of multiple local SSQ minima, contribute to the uncertainty of the analysis and need to be taken into account for a meaningful estimate of absolute uncertainties. Both contributions are included in the $95 \%$ confidence interval we provide for each fit parameter (ESI $\dagger$ ). Contrary to previous findings in the literature ${ }^{21,24}$, the example in Fig. 2 clearly shows that it is not possible to determine the absolute particle radius better than to within several nanometers if correlations and local minima are properly taken into account.

Fig. 3 illustrates the influence of the wavelength range included in the fit on the uncertainties of the absolute values of $R$ and $m(\lambda)$. The experimental BLS spectrum $\left(C_{\text {exp }}\right)$ is compared with two calculated cross-sections as a function of wavelength: $\mathrm{C}_{\text {sim,450-500 (orange }}$ trace) represents the best fit over a reduced wavelength interval $\lambda \epsilon[450,500] \mathrm{nm}$ and

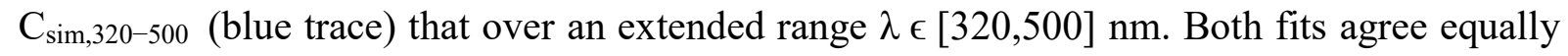
well with the experiment in the narrow wavelength interval between 450 and $500 \mathrm{~nm}$, especially with respect to the resonance peak positions. However, as Table 1 reveals, the resulting fit parameters are very different for the two intervals. As discussed in the previous subsection, such multiple sets of parameters with similar agreement with the measurement are the result of multiple local minima with similar SSQ values. The number of such multiple minima strongly increases for narrower wavelength intervals, which in turn leads to higher uncertainties in the absolute values of the parameters. In our example, the absolute uncertainties in $\mathrm{R}, \mathrm{m}_{0}$ and $\mathrm{m}_{1}$ are one order of magnitude higher when the fit is performed only over $\lambda \epsilon[450,500] \mathrm{nm}$ instead of $\lambda \epsilon[320,500] \mathrm{nm}$ (see Table 1). This example demonstrates that a broad wavelength range is needed to determine the radius within several nanometers and the refractive index within $\sim 0.01$ (for small particles). Good agreement between calculated and experimental scattering cross sections over a narrow wavelength interval is not sufficient to determine accurate particle radii.

\subsection{Reference wavelength, finite Cauchy expansion, imaginary refractive index}

Instead of Eq. (1), other parameterizations of the dispersion relation are often used without an explicit reference wavelength $\lambda_{0}\left(m\left(\lambda_{0}\right)=m_{0}\right)$, e.g. example $m(\lambda)=A+B / \lambda^{2}+C / \lambda^{4} 18$. Within the numerical accuracy all parameterizations evidently yield the same $m(\lambda)$. 
Statistically, however, they are not equivalent (ESI $\uparrow$, Section B.2). We find that introducing a finite reference wavelength, i.e. inclusion of $/{ }_{0}^{2}$ and $/{ }_{0}^{4}$ in the non-dispersive part, reduces the contribution of the dispersive parameters and thus the statistical uncertainty of $\mathrm{m}(\lambda)$. We choose $\lambda_{0}$ in the middle of the wavelength interval to minimize the factors $\left(\lambda_{0}^{2} / \lambda^{2}-1\right)$ and $\left(\lambda_{0}^{4} / \lambda^{4}-1\right)$ and hence the contribution of the dispersive part of $m(\lambda)$.

Two parameters ( $m_{0}$ and $m_{1}$ in Eq.(1)) are commonly used to describe the dispersion of the refractive index over relatively narrow wavelength ranges. Including higher order terms introduces more flexibility to improve agreement between simulation and experiment. We have tested the effect of including of a fourth order Cauchy term ( $\mathrm{m}_{2}$ in Eq.(1)) on the best fit parameters for a PSL sphere with a radius of $\mathrm{R}=1.5 \pm 0.15 \mu \mathrm{m}$ over the wavelength range $\lambda \in[320,500] \mathrm{nm}$. Compared with the case $\mathrm{m}_{2}=0$, refining $\mathrm{m}_{2}$ changes $\mathrm{R}$ by only $2 \mathrm{~nm}$, well within our estimated uncertainty. As expected $m(\lambda)$ changes slightly at the edges of the wavelength interval $(\sim 0.005)$ than closer to $\lambda_{0}(\sim 0.002)$, but with an average change of only 0.003 - about a third of the absolute uncertainty - the dispersion of $m(\lambda)$ over the wavelength range of our broad band measurements is still accurately described by a second order Cauchy expansion.

For strongly absorbing particles, the inclusion of the imaginary part $\mathrm{k}$ of the refractive index is essential to obtain reliable results from $\operatorname{BLS} . m(\lambda)$ and $k(\lambda)$ are coupled through the Kramer-Kronig relation ${ }^{34}$, which needs to be taken into account when both parameters $m(\lambda)$ and $\mathrm{k}(\lambda)$ are fitted. Moreover, Eq. (1) becomes invalid if $\mathrm{k}(\lambda)$ changes substantially over a narrow wavelength range (i.e. in the region of a strong absorption) and $m(\lambda)$ needs to be expressed in a different way. The samples of the present study are non- or only very weakly absorbing. A non-zero $\mathrm{k}$ broadens the resonances and decreases their maximum amplitudes. Our simulations show that with the $1.1 \mathrm{~nm}$ resolution of the spectrometer used in this study, $\mathrm{k}$ values below $10^{-3}$ have a negligible effect on the extinction cross sections compared with the influence of the spectrometer resolution. For this reason, we assume $k(\lambda)=0$ in the following.

\section{Case studies}

\subsection{Sizing of submicron PSL spheres}

BLS experiments in the VIS range are restricted to the characterization of micron-sized aerosol particles ${ }^{21,22,24,25}$. Here, we present several applications of our BLS setup that exploit 
the possibility to size submicron particles by using broadband radiation in the UV spectral range. First we test our approach with calibrated micron-sized PSL beads, which have been extensively used as reference particles for sizing ${ }^{21,35,36}$. Two main issues arise in this context. The indicated refractive index of PLS beads is usually not calibrated and literature measurements of the refractive index of different samples of size-calibrated PSL microspheres at the same wavelengths are spread over a broad range from 1.587 to 1.626 (for $\lambda=560 \mathrm{~nm})^{21}$. Even if the same measurement technique and micro-beads from the same PLS sample are used, the retrieved refractive index has still a standard deviation higher than $0.002^{21}$. In addition, the absolute determination of $\mathrm{R}$ and $\mathrm{m}(\lambda)$ for PLS beads is impaired by surface modifications due to the unavoidable coating with residual ligands after nebulization and drying $( \pm 0.4 \mathrm{~nm})^{36}$, by inhomogeneity of the beads, and by their asphericity $(0.6 \%)^{36}$. Keeping these limitations in mind - and without better alternatives - PSL beads are still useful for testing particle sizing techniques.

Here, we use NIST calibrated PSL submicron-beads specified with $\mathrm{R}=509.5 \pm 7.5 \mathrm{~nm}$ and $\mathrm{m}(\lambda=589 \mathrm{~nm})=1.59$ to validate the performance of our BLS experiment for the sizing of submicron particles. The NIST size calibration is based on photon correlation spectroscopy and transmission electron microscope measurements. Fig. 4 compares the measured BLS with the corresponding best fit between $320 \mathrm{~nm}$ and $500 \mathrm{~nm}$ for an individual submicron-bead. The noise in the experimental spectrum is due to the comparatively low light intensity in the UV region and the low scattering efficiency of small particles. It can be reduced by using longer averaging times and a more intense UV light source. The noise notwithstanding, measurement and fit are in very good agreement regarding not only the positions of the resonances but also their intensity profiles. We derive a particle size of $R=515 \mathrm{~nm}$ with $\Delta_{R}=[511.2,518.8] \mathrm{nm}$ and a refractive index of $\mathrm{m}(\lambda=589 \mathrm{~nm})=1.587$ with $\Delta_{\mathrm{m}(\lambda=589 \mathrm{~nm})}=[1.568,1.606]$ which both agree with the NIST specifications. The wavelength-dependence of $m(\lambda)$ can be calculated from the fitted Cauchy parameters provided in the figure caption. It agrees with the wavelength-dependence measured by Jones et al. ${ }^{21}$ in the region where the two experiments overlap, i.e. between $480 \mathrm{~nm}$ and $500 \mathrm{~nm}$. The same holds for the spread of $\mathrm{m}(\lambda)$ observed for different particles. The BLS spectrum of the $515 \mathrm{~nm}$ PSL sphere shown in Fig. 4 features 12 resonances between $320 \mathrm{~nm}$ and $500 \mathrm{~nm}$. Particles can be sized with fewer resonances, especially when the full spectrum is fitted and not only the peak positions. We estimate a minimum of four resonances for a reliable size determination, from which we conclude that PSL beads as small as $300 \mathrm{~nm}$ can be accurately sized with our experimental setup. 


\subsection{Evaporation of a binary glycerol water droplets}

BLS measurements are attractive for evaporation and growth studies of multicomponent aerosol particles because information on both the vapour pressure and the droplet composition can be obtained simultaneously through the measurement of the droplet size and wavelengthdependent refractive index. As an example, we perform here evaporation experiments for glycerol water droplets at a constant water relative humidity $(\mathrm{RH})$ of $50 \pm 3 \%$ at a temperature of $293 \mathrm{~K}$, corresponding to a glycerol mass fraction of $83.0 \pm 2.5 \%{ }^{37}$. If partial equilibrium with respect to water is maintained during evaporation the composition of the droplet must remain constant because by virtue of its chemical potential the mass fraction of water in the droplet is fixed at a fixed RH.

Fig. 5 presents the temporal behaviour of the droplet radius $\mathrm{R}$ and the wavelengthdependent refractive index as obtained from the BLS fit. The decrease in R corresponds to a decrease in the total droplet volume of $82 \%$. As expected, $\mathrm{R}^{2}$ decreases linearly with time (see Fig. B in the ESI $\dagger$ ) with a slope of $-3.34 \times 10^{-15} \mathrm{~m}^{2} \cdot \mathrm{s}^{-1}$. This slope is in good agreement with the slope of $-3.55 \times 10^{-15} \mathrm{~m}^{2} \cdot \mathrm{s}^{-1}$ determined previously for large glycerol water droplets at $50 \% \mathrm{RH}^{37}$. The wavelength-dependence of $\mathrm{m}(\lambda)$ is shown in the lower trace of Fig. 5 . As can be seen it systematically decreases with increasing wavelength. The temporal variation of $m$ is indicated by the standard deviation over time for each $\lambda$ (shaded area). It clearly shows that to a good approximation the composition of the droplet stays constant over time in agreement with the results from ref. ${ }^{37}$. For example, the mean value of the refractive index and its standard deviation at $\lambda=589 \mathrm{~nm}$ amount to $\mathrm{m}(\lambda=589 \mathrm{~nm})=1.4495 \pm 0.0022$, which corresponds to a mean value in the glycerol mass fraction of $84.0 \pm 1.5 \%{ }^{38}$. The standard deviation lies well within the uncertainty of the mass fraction retrieved from the $\mathrm{RH}$ measurement $( \pm 2.5 \%)$. A refractive index of $\mathrm{m}(\lambda=589 \mathrm{~nm})=1.4495 \pm 0.0022$ agrees well with previous literature data of $\mathrm{m}(\lambda=589 \mathrm{~nm})=1.4493$ corresponding to $50 \% \mathrm{RH}^{38}$.

The vapour pressure of a pure liquid can be determined from the slope of $\mathrm{R}^{2}$ recorded during the evaporation of a pure droplet if the gas phase diffusion coefficient is known ${ }^{39}$. With the composition kept constant at constant RH this approach can be analogously applied to our binary glycerol-water mixture, using the gas phase diffusion coefficient of glycerol $\left(8.77 \times 10^{-6} \mathrm{~m}^{2} \cdot \mathrm{s}^{-1} 40\right)$, the glycerol mass fraction of the droplet $(83.0 \pm 2.5 \%)$, and the activity coefficient of glycerol corresponding to this mixture $\left(0.937 \pm 0.014{ }^{40}\right){ }^{37}$. This results in a 
glycerol vapour pressure of $p_{\text {glyc }}=11.07 \pm 0.68 \mathrm{mPa}$, which lies very close to value of $p_{\text {glyc }}=11.6 \mathrm{mPa}$ that was determined for pure bulk glycerol ${ }^{41}$.

\subsection{Hygroscopic growth and shrinkage of aqueous $\mathrm{K}_{2} \mathrm{CO}_{3}$ droplets}

The Köhler theory describes the cloud condensation nuclei (CCN) activity of an aerosol particle by parameterizing solute effects using a hygroscopicity parameter $\kappa^{12}$. The CCN activity as a function of $\mathrm{RH}$ can be derived directly from $\kappa$, thus providing information on cloud formation and the particle's indirect effect on climate $^{42} . \kappa$ can be determined from the droplet radius measured as a function of the $\mathrm{RH}$ in hygroscopic growth and shrinkage experiments of single aerosol particles (see eq. (6) of ref. ${ }^{12}$ ) assuming that equilibrium is established. Fig. 6b shows the droplet radius R determined as a function of the RH (Fig. 6a) from BLS experiments of single aqueous $\mathrm{K}_{2} \mathrm{CO}_{3}$ droplets. $\mathrm{R}$ nicely follows the changes in RH. From these measurements, we derive a hygroscopicity parameter $\kappa_{K_{2} \mathrm{CO}_{3}}=1.07 \pm 0.10$, which agrees with the previously determined value of $\kappa_{K_{2} \mathrm{CO}_{3}}=0.90$ from ref. ${ }^{43}$.

In addition to the hygroscopicity parameter our BLS measurements provide concentration and wavelengths dependent refractive indices (Figs. 6c and d). $\mathrm{m}_{0}$ governs the concentration dependence of $m(\lambda)$, while the small dispersive contributions remain constant apart from insignificant fluctuations in $\mathrm{m}_{1}$ that mainly arise from noise in the BLS measurements. $\mathrm{R}$ and $\mathrm{m}_{0}$ (and thus $\mathrm{m}(\lambda)$ ) follow opposite trends with changing droplet composition. Increasing $\mathrm{RH}$ lets the droplet grow ( $\mathrm{R}$, trace $\mathrm{b}$ ), decreasing the concentration of (non-volatile) $\mathrm{K}_{2} \mathrm{CO}_{3}$ and hence lowering the refractive index $\left(\mathrm{m}_{0}\right.$, trace $\left.\mathrm{c}\right)$. A comparison with literature values for $\mathrm{m}(\lambda)$ (only available for $\lambda=589 \mathrm{~nm}$ at $\mathrm{T}=293 \mathrm{~K}$ ) at different concentrations in the range 4.4-9.2 $\mathrm{mol} / \mathrm{kg} 44$ is complicated by the fact that the data needed to calculate the droplet concentrations (see ref. ${ }^{12}$ and ${ }^{45}$ ) are only provided at higher temperatures (298 K instead of 293K). In addition, the data from ${ }^{45}$ and ${ }^{44}$ had to be extrapolated, because the water activity and $\mathrm{K}_{2} \mathrm{CO}_{3}$ concentration in our measurements are outside of the ranges considered in these references. Furthermore, $\lambda=589 \mathrm{~nm}$ lies outside the range of wavelengths we used for the $\mathrm{K}_{2} \mathrm{CO}_{3}$ BLS measurements $(320-500 \mathrm{~nm})$. Finally, the accuracy of our RH probes is currently limited to $\pm 3 \%$. The values we calculate for $\mathrm{m}(\lambda=589 \mathrm{~nm})$ lie slightly above the literature values, by about 0.01 , a reasonable agreement considering the above-mentioned uncertainties. The systematic shift might be explained by the difference in temperature compared with ref. ${ }^{45}$. 


\subsection{Photochemistry of oleic acid droplets}

The study of photochemical reactions with aerosol particles is of key importance for the understanding of aerosol ageing and its impact on both health and climate ${ }^{46}$. In our final example we suggest using the UV part of the broadband light source to induce photochemical reactions in oleic acid droplets while at the same time extracting particle size and refractive index from the BLS measurements. The oxidative aging of droplets containing oleic acid in the presence of ozone has previously been investigated ${ }^{24}$. The oxidation of the oleic acid particle was identified by a decrease of the particle's radius $\mathrm{R}$ due to the evaporation of volatile oxidation products and an increase of the particle's refractive index. Here, we use UV light to induce photolysis of oleic acid (purity higher than $99 \%$ ) droplets in the absence of ozone and BLS measurements to determine $\mathrm{R}$ and $\mathrm{m}(\lambda)$ to follow the progress of the reaction. The determination of the mechanism of the photochemical reactions is beyond the scope of this paper. To avoid the formation of ozone from $\mathrm{O}_{2}$, which can occur for $\lambda \leq 242 \mathrm{~nm}^{47}$, we flushed the trapping cell with a continuous dry $\mathrm{N}_{2}$ flow of 0.4 standard cubic centimeter (scc) per minute replacing the gas in the cell volume (approximately $1.5 \mathrm{scc}$ ) in a few minutes.

Fig. 7 shows typical experimental results. The trapped particle was first equilibrated in the trapping cell for 15 minutes with the light source turned off (not shown). Then, the light source was turned on for $\sim 15$ seconds during which BLS measurements were taken (squares at $\mathrm{t}=0 \mathrm{~s}$ in Fig. 7). After that, the source was turned off again for $\Delta \mathrm{t}=10$ minutes. This cycle was repeated four times (first four squares with four intervals $\Delta \mathrm{t}$ ). Even though the UV light was on for only a few seconds the droplet radius (trace a) decreases noticeably a drop with time and $m_{0}$ shows a small increase (trace $b$ ), which are first indications of photochemical reactions occurring. After the four cycles (at $t \sim 2500 \mathrm{~s}$ ) the light source is turned on for approximately 25 minutes until the particle escapes from the trap (at $\mathrm{t} \sim 4000 \mathrm{~s}$ ). During this period of continuous light exposure $\mathrm{R}$ decreases by a factor of two due to the evaporation of volatile oxidation products. This is accompanied by a pronounced increase of $m(\lambda)$ and its dispersion $\left(\mathrm{m}_{0}\right.$ and $\left.\mathrm{m}_{1}\right)$ probably due to the formation of non-volatile reaction products. Just before the escape of the particle, the fluctuations in $\mathrm{R}, \mathrm{m}_{0}$, and $\mathrm{m}_{1}$ increase. $\mathrm{m}_{1}$ is more affected by these fluctuations than $\mathrm{m}_{0}$ and in particular $\mathrm{R}$, for which the fluctuations are barely visible. The fluctuations might arise from an increasing instability of the particle in the trap. Similar measurements have been performed for six particles, which all showed the same behaviour. The smallest oleic acid particle we could measure had a radius of $\mathrm{R}=390.5 \mathrm{~nm}$ with $\Delta_{\mathrm{R}}=[381.0,400.0] \mathrm{nm}$ (see Fig. $\mathrm{C}$ in the ESI $\dagger$ ). The limiting factor for 
the smallest particle size detectable is not the BLS sensitivity but the trapping stability of the oxidized particle. These measurements demonstrate that UV photochemistry can be induced by the broadband radiation and simultaneously detected by BLS. For a more detailed analysis of the refractive index change, it will be essential to include the determination of the imaginary part of the refractive index as briefly addressed in subsection 3.5.

\section{Summary}

We present a broadband light scattering (BLS) setup for the characterization of single optically-trapped aerosol particles covering a very broad wavelengths range including the ultraviolet (UV) region. The inclusion of the UV range allows us to accurately size submicron-sized aerosol particles down to radii of about $300 \mathrm{~nm}$. Furthermore, the extension into the UV provides refractive index data of aerosol particles over a very broad wavelength range from 320 to $700 \mathrm{~nm}$. Wavelength-dependent refractive index data of aerosol particles are important, for example to assess their climate impact; but they are hard to determine and thus rarely available, in particular in the UV range.

The important parameters that determine the uncertainty in the absolute values of the particle radius and the particle refractive index are the correlations between the fit parameters and the wavelength interval that is included in the fit. As a rough guideline, a wavelength interval of more than $150 \mathrm{~nm}$ is required to determine the particle radius to within several nanometers and the refractive index to within 0.01 . The uncertainties in relative changes of the radius and the refractive index are approximately one order of magnitude lower than the quoted absolute uncertainties. We have demonstrated the performance of the UV BLS setup for various applications. They include sizing of submicrometer particles, monitoring of photochemical reactions, and evaporation and growth studies of binary mixtures, which allow us to determine the vapour pressure of low-volatile compounds as well as the properties of aerosol particles as cloud condensation nuclei. These examples demonstrate main advantage of the UV BLS setup, viz. its broad applicability, for which the extension to submicron-sized particles is crucial.

\section{Acknowledgements}


This work was supported by the Swiss National Science Foundation (SNSF grant nr. 200020_159205) and ETH Zurich. We would like to acknowledge the contributions of Johannes Cremer, Gian-Marco Schnüriger, and Gianluca Janka in the early stage of this projects. 


\section{Figures}

Fig. 1: Schematic of the BLS setup (see text).

Fig. 2: Two dimensional cuts through the SSQ as a function of the fit parameters. (a) R and $\mathrm{m}_{0}$, (b) $\mathrm{m}_{1}$ and $\mathrm{m}_{0}$, and (c) $\mathrm{R}$ and $\mathrm{m}_{1}$. The third parameter for each map is set to its value corresponding to best fit $\left(\mathrm{R}=1405 \mathrm{~nm}, \mathrm{~m}_{0}=1.656, \mathrm{~m}_{1}=0.1124\right.$, respectively). The color code indicates the SSQ value (arbitrary units). The white circles, located in the middle of the dark blue regions, show the optimal set of parameters. The $95 \%$ confidence intervals for this fit are $\Delta_{\mathrm{R}}=[1393,1413] \mathrm{nm}, \Delta_{\mathrm{m} 0}=[1.6454,1.6714]$ and $\Delta_{\mathrm{m} 1}=[0.1079,0.1171]$.

Fig. 3: Black line: Experimental scattering cross-section $\mathrm{C}_{\exp }$ for a size-calibrated $1.5 \pm 0.15 \mu \mathrm{m}$ PSL sphere from BLS measurements. Calculated scattering cross-sections $\mathrm{C}_{\text {sim }}$ determined using the wavelength intervals $\lambda \in[450,500] \mathrm{nm}$ (orange line) and $\lambda \in[320,500] \mathrm{nm}$ (blue line), respectively. The two intervals are indicated by vertical dotted lines of the respective colors.

Fig. 4: Experimental BLS spectrum $\left(\mathrm{C}_{\exp }\right)$ recorded for NIST calibrated PLS beads of a radius of $\mathrm{R}=509.5 \pm 7.5 \mathrm{~nm}$ radius. The spectrum was averaged over $2 \mathrm{~s}$. Best fit $\left(\mathrm{C}_{\text {sim,320-500 }}\right)$ providing the following parameters with $95 \%$ coincidence intervals: $\mathrm{R}=515 \mathrm{~nm}$ with $\Delta_{\mathrm{R}}=[511.2, \quad 518.8] \mathrm{nm}, \quad \mathrm{m}_{0}=1.633$ at $\lambda_{0}=400 \mathrm{~nm} \quad$ with $\Delta_{\mathrm{m} 0}=\left[\begin{array}{ll}1.618, & 1.648\end{array}, \quad\right.$ and $\mathrm{m}_{1}=0.0848$ with $\Delta_{\mathrm{m} 1}=[0.0778,0.918]$. See also Eq. (1) and the ESI $\uparrow$.

Fig. 5: Evaporation of glycerol water droplets at 50\% RH. Top panel: Time evolution of the droplet radius R. Bottom panel: Wavelength-dependence of the refractive index $m(\lambda)$. The temporal variation of $\mathrm{m}$ is indicated by its standard deviation (shaded area) for each $\lambda$.

Fig. 6: Hygroscopic growth and shrinkage of aqueous $\mathrm{K}_{2} \mathrm{CO}_{3}$ droplets. (a) Time evolution of the RH. (b) Time evolution of the droplet radius R. (c) and (d) Time evolution of the Cauchy parameters $\mathrm{m}_{0}$ and $\mathrm{m}_{1}$, respectively, $\left(\lambda_{0}=400 \mathrm{~nm}\right.$ and $\mathrm{m}_{2}=0$, see Eq. (1)).

Fig 7: Photochemistry of oleic acid droplets induced by UV light and detected by BLS measurements (wavelength range 320-500 nm). (a) Time evolution of the droplet size $\mathrm{R}$ (top 
panel). (b) and (c) Time evolution of the Cauchy parameters $m_{0}$ and $m_{1}$, respectively, $\left(\lambda_{0}=400 \mathrm{~nm}\right.$ and $\mathrm{m}_{2}=0$, see Eq.(1)). $\Delta \mathrm{t}$ indicates the times during which the broadband light source was turned off. 


\section{Table}

Table 1: Comparison of fit results for the same particle (PSL sphere, $R=1550 \pm 145 \mathrm{~nm}$ ) using two different wavelength ranges $[\lambda]$ in the fit. Optimal parameters $R, m_{0}$, and $m_{1}$ and their $95 \%$ confidence interval $\left(\Delta_{\mathrm{R}}, \Delta_{\mathrm{m} 0}\right.$ and $\Delta_{\mathrm{m} 1}$, respectively). For the [450,500] nm wavelength range, $\Delta_{\mathrm{R}}, \Delta_{\mathrm{m} 0}$ and $\Delta_{\mathrm{m} 1}$ are broad and asymmetric because three local minima contribute. The three minima are characterized by the following ranges: $\mathrm{R} \in[1328,1347] \mathrm{nm},[1570,1603]$ $\mathrm{nm}, \quad[2847,3008] \mathrm{nm}, \quad \mathrm{m}_{0} \in[1.633,1.6724], \quad[1.346,1.38], \quad[1.582,1.636]$ and $\mathrm{m}_{1} \in[0.1207,0.1447],[0.0608,0.0817]$, [0.0798,0.981].

\begin{tabular}{|c|c|c|c|c|c|c|c|}
\hline$[\lambda](\mathrm{nm})$ & $\lambda_{0}(\mathrm{~nm})$ & $\mathrm{R}(\mathrm{nm})$ & $\Delta_{\mathrm{R}}(\mathrm{nm})$ & $\mathrm{m}_{\mathrm{o}}$ & $\Delta_{\mathrm{m} 0}$ & $\mathrm{~m}_{1}$ & $\Delta_{\mathrm{m} 1}$ \\
\hline$[320,500]$ & 400 & 1406.5 & {$[1394.0,1419.0]$} & 1.656 & {$[1.6396,1.6724]$} & 0.1131 & {$[0.1068,0.1196]$} \\
\hline$[450,500]$ & 475 & 1586.0 & {$[1328.0,3008.0]$} & 1.3632 & {$[1.3436,1.6584]$} & 0.0703 & {$[0.0608,0.1443]$} \\
\hline
\end{tabular}




\section{References}

1. C. F. Bohren and D. R. Huffman, Absorption and Scattering of Light by Small Particles, Wiley, New York, 1983.

2. $\quad$ S. J. Ghan and S. E. Schwartz, Bull. Am. Meteorol. Soc., 2007, 88, 1059-1083.

3. J. Haywood and O. Boucher, Rev. Geophys., 2000, 38, 513-543.

4. M. I. Mishchenko, L. D. Travis and A. A. Lacis, Scattering, Absorption, and Emission of Light by Small Particles, Cambridge university press, UK, 2002.

5. M. Kahnert, T. Nousiainen and H. Lindqvist, Opt. Express, 2013, 21, 7974-7993.

6. T. Sakai, T. Nagai, Y. Zaizen and Y. Mano, Appl. Opt., 2010, 49, 4441-4449.

7. M. Goldmann, J. Miguel-Sánchez, A. H. C. West, B. L. Yoder and R. Signorell, J. Chem. Phys., 2015, 142, 224304.

8. G. David, K. Esat, S. Hartweg, J. Cremer, E. Chasovskikh and R. Signorell, J. Chem. Phys., 2015, 142, 154506.

9. T. Nishizawa, N. Sugimoto, I. Matsui, A. Shimizu and H. Okamoto, J. Quant. Spectrosc. Radiat. Transfer, 2011, 112, 254-267.

10. A. Miffre, G. David, B. Thomas and P. Rairoux, Geophys. Res. Lett., 2011, 38, L16804.

11. M. I. Cotterell, B. J. Mason, A. E. Carruthers, J. S. Walker, A. J. Orr-Ewing and J. P. Reid, Phys. Chem. Chem. Phys., 2014, 16, 2118-2128.

12. M. D. Petters and S. M. Kreidenweis, Atmos. Chem. Phys., 2007, 7, 1961-1971.

13. M. D. King, K. C. Thompson, A. D. Ward, C. Pfrang and B. R. Hughes, Faraday Discuss., 2008, 137, 173-192.

14. R. E. H. Miles, A. E. Carruthers and J. P. Reid, Laser Photonics Rev., 2011, 5, 534-552.

15. M. D. King, K. C. Thompson and A. D. Ward, J. Am. Chem. Soc., 2004, 126, 16710-16711.

16. J. D. Eversole, H. B. Lin, A. L. Huston, A. J. Campillo, P. T. Leung, S. Y. Liu and K. Young, J. Opt. Soc. Am. B, 1993, 10, 1955-1968.

17. A. A. Zardini, U. K. Krieger and C. Marcolli, Opt. Express, 2006, 14, 6951-6962.

18. A. D. Ward, M. Zhang and O. Hunt, Opt. Express, 2008, 16, 16390-16403.

19. M. I. Cotterell, B. J. Mason, T. C. Preston, A. J. Orr-Ewing and J. P. Reid, Phys. Chem. Chem. Phys., 2015, 17, 15843-15856.

20. R. A. Washenfelder, J. M. Flores, C. A. Brock, S. S. Brown and Y. Rudich, Atmos. Meas. Tech., 2013, 6, 861-877.

21. S. H. Jones, M. D. King and A. D. Ward, Phys. Chem. Chem. Phys., 2013, 15, 20735-20741.

22. L. J. Moore, M. D. Summers and G. A. D. Ritchie, Phys. Chem. Chem. Phys., 2013, 15, 1348913498.

23. M. Guillon and B. Stout, Phys. Rev. A, 2008, 77, 023806.

24. T. C. Preston and J. P. Reid, J. Opt. Soc. Am. B, 2013, 30, 2113-2122.

25. S. H. Jones, M. D. King and A. D. Ward, Chem. Commun., 2015, 51, 4914-4917.

26. Y. J. Kaufman, D. Tanre and O. Boucher, Nature, 2002, 419, 215-223.

27. H. R. Pruppacher and J. D. Klett, Microphysics of clouds and precipitation, Wiley Online Library.

28. IPCC, 2014: Climate Change 2014: Synthesis Report. Contribution of Working Groups I, II and III to the Fifth Assessment Report of the Intergovernmental Panel on Climate Change [Core Writing Team, R.K. Pachauri and L.A. Meyer (eds.)]. IPCC, Geneva, Switzerland, 151 pp.

29. I. Thanopulos, D. Luckhaus, T. C. Preston and R. Signorell, J. Appl. Phys., 2014, 115, 154304.

30. T. Li, S. Kheifets, D. Medellin and M. G. Raizen, Science, 2010, 328, 1673-1675.

31. L. Rkiouak, M. J. Tang, J. C. J. Camp, J. McGregor, I. M. Watson, R. A. Cox, M. Kalberer, A. D. Ward and F. D. Pope, Phys. Chem. Chem. Phys., 2014, 16, 11426-11434.

32. C. C. Lam, P. T. Leung and K. Young, J. Opt. Soc. Am. B, 1992, 9, 1585-1592.

33. J. Li, S.-T. Wu, S. Brugioni, R. Meucci and S. Faetti, J. Appl. Phys., 2005, 97, 073501. 
34. V. Lucarini, Kramers-Kronig relations in optical materials research, Springer Science \& Business Media, 2005.

35. D. T. Phillips, P. J. Wyatt and R. M. Berkman, J. Colloid Interface Sci., 1970, 34, 159-162.

36. G. W. Mulholland, A. W. Hartman, G. G. Hembree, E. Marx and T. R. Lettieri, J. Res. Nat. Bur Stand., 1985, 90, 3-26.

37. A. K. Ray, R. D. Johnson and A. Souyri, Langmuir, 1989, 5, 133-140.

38. L. F. Hoyt, Ind. Eng. Chem., 1934, 26, 329-332.

39. I. N. Tang and H. R. Munkelwitz, J. Colloid Interface Sci., 1991, 141, 109-118.

40. A. Kiraz, Y. Karadag and M. Muradoglu, Phys. Chem. Chem. Phys., 2008, 10, 6446-6454.

41. H. K. Cammenga, F. W. Schulze and W. Theuerl, J. Chem. Eng. Data, 1977, 22, 131-134.

42. M. Kanakidou, J. H. Seinfeld, S. N. Pandis, I. Barnes, F. J. Dentener, M. C. Facchini, R. Van Dingenen, B. Ervens, A. Nenes, C. J. Nielsen, E. Swietlicki, J. P. Putaud, Y. Balkanski, S. Fuzzi, J. Horth, G. K. Moortgat, R. Winterhalter, C. E. L. Myhre, K. Tsigaridis, E. Vignati, E. G. Stephanou and J. Wilson, Atmos. Chem. Phys., 2005, 5, 1053-1123.

43. P. Kumar, I. N. Sokolik and A. Nenes, Atmos. Chem. Phys., 2011, 11, 8661-8676.

44. W. M. E. Haynes, CRC handbook of chemistry and physics, CRC press., 2015.

45. M. Sarbar, A. K. Covington, R. L. Nuttall and R. N. Goldberg, J. Chem. Thermodyn. Thermochem., 1982, 14, 695-702.

46. C. George, M. Ammann, B. D’Anna, D. J. Donaldson and S. A. Nizkorodov, Chem. Rev., 2015, $115,4218-4258$.

47. R. Atkinson, D. L. Baulch, R. A. Cox, J. N. Crowley, R. F. Hampson, R. G. Hynes, M. E. Jenkin, M. J. Rossi and J. Troe, Atmos. Chem. Phys., 2004, 4, 1461-1738. 